\title{
Hemodynamics in Prefrontal and Temporal Cortex during Attentional Control Task*
}

\author{
Kanako MiKAmI $^{\dagger}$ and Kunihiko OURA ${ }^{\ddagger}$
}

\begin{abstract}
This paper discusses hemodynamics in both the prefrontal and temporal cortex when subjects taking in glucose perform the Paced Auditory Serial Addition Test (PASAT). PASAT can greatly stress the working memory associated with attention. Attention is well known as the base of various higher-order cognitive functions. Some studies evaluate how the auditory attentional function was activated using fMRI during Clinical Assessment for Attention (CAT), but few reports use Near-infrared Spectroscopy NIRS. In previous research studies, the authors reported that oxidized hemoglobin concentration $\mathrm{HbO}$ increased in the left DLPFC area during PASAT. In this study, two types of NIRS were used to evaluate $\mathrm{HbO}$ for both the prefrontal and temporal cortex. Multivariable estimated models of auto-regressive form with upper triangular estimates are applied to analyze the results from the experiment. The models are given by hierarchical decomposition analysis which shows the properties of each stage.
\end{abstract}

\section{Introduction}

Recently, Near-Infrared Spectroscopy (NIRS) has been used in various fields including psychiatry and cognitive rehabilitation for its usefulness in diagnosing mental disorders such as depression [1]. Higherorder brain dysfunction is a disorder that appears after cerebral damage, often caused by injury. Attention disorder is one of the more serious troubles related to various higher-order cognitive functions. The Paced Auditory Serial Addition Test (PASAT) developed by Gronwall to evaluate and judge attention disorders in neuropsychology. It is included in Clinical Assessment for Attention (CAT) a professional standard issued in 2006 by the Japan Society for Higher Brain Dysfunction.

Attention is well known as the base of various higher-order cognitive functions. Generally, attention is defined as a multidimensional process that enables the brain to focus on incoming stimuli. This process

\footnotetext{
* Manuscript Received Date: June 24, 2016

The material of this paper was partially presented at the 47th ISCIE International Symposium on Stochastic Systems Theory and Its Applications (SSS'15) which was held in Dec., 2015.

$\dagger$ Graduate School of Engineering, Kokushikan University; 4-28-1, Setagaya, Setagaya-ku, Tokyo 154-8515, JAPAN

¥ School of Science and Engineering, Kokushikan University; 4-28-1, Setagaya, Setagaya-ku, Tokyo 154-8515, JAPAN

Key Words: hemodynamics, cerebral cortex, NIRS, PASAT, attentional function.
}

is based on components of alertness or readiness to respond and vigilance or the capacity to select relevant stimuli needed for conscious processing [2]. Sohlberg and Mateer's attention process model divides attention into a hierarchy of five levels: focused, sustained, selective, alternating and divided (Fig. 1). In the process of cognitive control by attention, the brain keeps necessary information temporarily and addressed the problem. This process is related to function included in working memory and located in Broadmann Area 9 and 46.

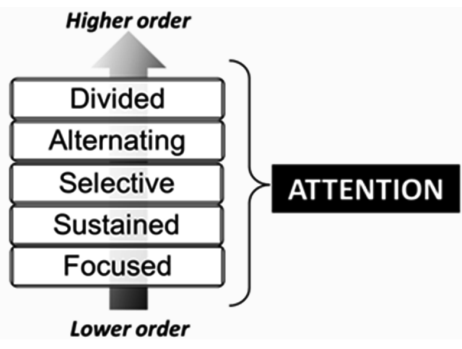

Fig. 1 The classification of attentional function

Although there have been some studies on brain activities measured by functional Magnetic Resonance Imaging (fMRI) during PASAT (e.g. Sakai) [3], there are few studies on measuring brain activity using NIRS NIRS is effective for this purpose as it can measure brain activities safely, easily and noninvasively.

Glucose is an important energy source for brain activity. In this paper, the authors examined the influence of increased blood sugar levels on attention. Oxygen and glucose are used for nerve activities. The capillary reaction during this time is called neurovas- 
cular coupling (NVC) [4]. There is the connection between brain blood flow and glucose metabolism at rest in various parts of the cerebral cortex, as observed by autoradiography [5]. The amount of brain blood flow corresponds to glucose availability in the brain, as detected by positron emission tomography [6]. In light of these facts, the authors assume that it is necessary to evaluate glucose concentration in blood using an NVC -based experiment.

In this paper, we will show the results of the brain activities measured by two types of NIRS; the first measures frontal lobe activity (WOT-100) and the second measures temporal lobe activity (ETG-7000).

\section{Experimental Setup}

Subjects are tested after not eating for at least 6 hours. Subjects measure their own blood sugar level (BSL) three times every ten minutes by taking a blood sample. After the BSL data are obtained, the hemodynamics of the cerebral cortex is determined by NIRS. Each subject is instructed to verbally count upward beginning with "one" during this period. This procedure serves as a control test to determine the baseline of the subject's brain blood flow. Next, the subject performs the Pre-Test for warm-up, and then subject performs the PASAT as specified in the CAT. There is a one-minute intermission period between tests. In one of the two tests sequences, subject intakes $15[\mathrm{~g}]$ of glucose. The order of the test sequences is randomly determined. The experimental procedures are schematically shown in Fig. 2. The duration of the experiment is about eight minutes.

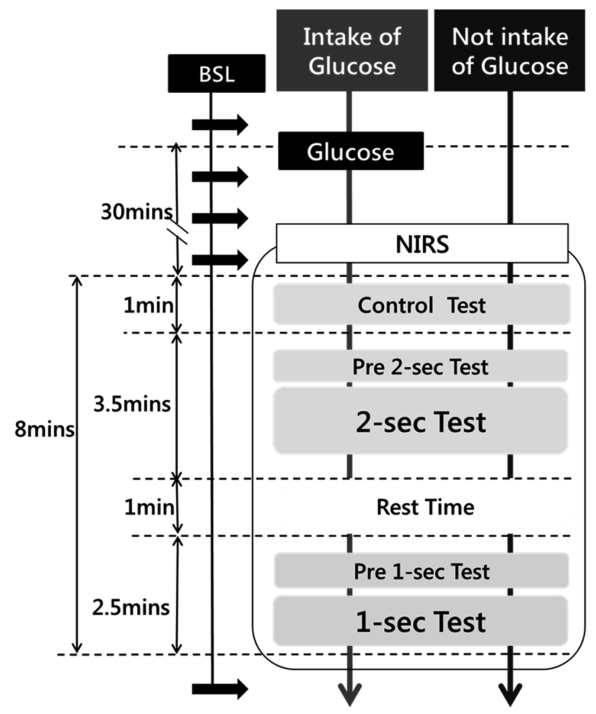

Fig. 2 The summaries of the experiment

The data is obtained from 14 healthy subjects (10 males and 4 females, age range 21 to 24 years).

All the subjects were informed, and they consented to all conditions of the experiment prior to start it. This study has been approved by the Ethics Committee for Human Research at Kokushikan University.
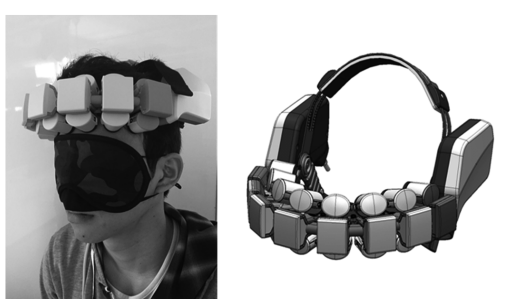

(a) A scene of the experiment and WOT-100 equipment

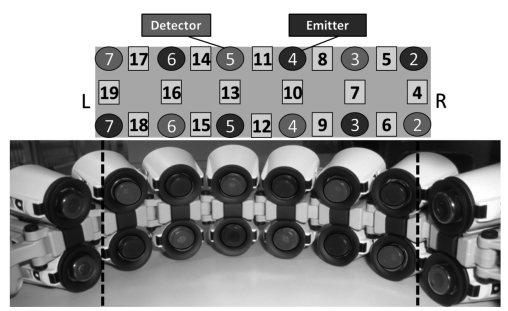

(b) The arrangement of 16 channels

Fig. 3 NIRS: Wearable Optical Topography System [WOT-100] by HITACHI

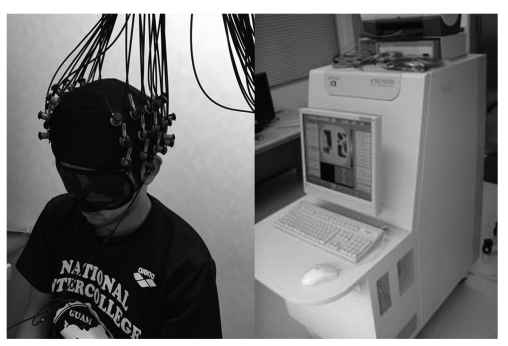

(a) A scene of the experiment and ETG-7000 equipment

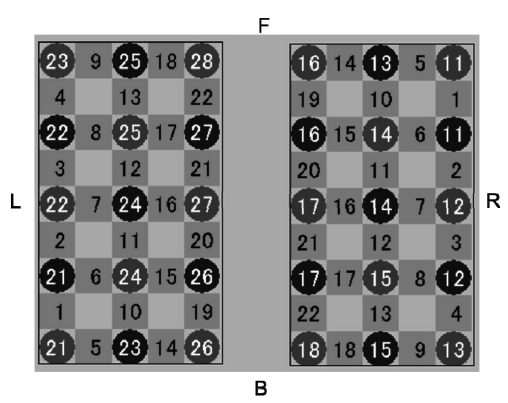

(b) The arrangement of 44 channels

Fig. 4 NIRS: Optical Topography System [ETG-7000] by HITACHI

\subsection{Near Infrared Spectroscopy}

NIRS is well known for its ability to observe brain activity hemodynamics. The NIRS equipment used in this experiment were a wearable optical topography system (WOT-100, Fig. 3) and an optical topography system (ETG-7000, Fig. 4), both manufactured by HITACHI. The concentrations of three kinds of hemoglobin, oxy-hemoglobin ( $\mathrm{HbO})$, deoxyhemoglobin $(\mathrm{HbR})$ and total-hemoglobin $(\mathrm{HbT})$ were 


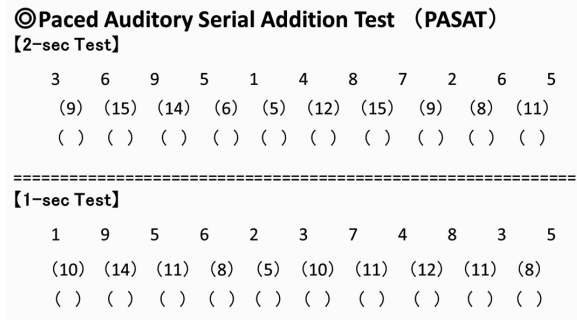

Fig. 5 Sample test paper of PASAT (Top: 2-sec Test, Bottom: 1-sec Test)

measured in the cerebral cortex. The WOT-100 has two rows of transmitters with each row having three emitters and three detectors in alternating order and the channels for observation are allocated between an emitter and a detector. Its sampling rate was pre-set at $200 \mathrm{~ms}$. The ETG-7000 has three rows with each row having five transmitters. As in the case of WOT100, the channels are placed between an emitter and a detector arranged in alternating order. Its sampling rate was set at $100 \mathrm{~ms}$. The test sequences were performed in a quiet room while the subjects wore probes on their head with an eye-mask and head-phones to enable their concentration on each task (Fig. 3 and Fig. 4).

Although there are three kinds of hemoglobin concentrations, the authors used only the time series data of $\mathrm{HbO}$ for analysis because $\mathrm{HbO}$ is considered to reflect brain activity, better than the other two [7].

\subsection{Paced Auditory Serial Addition Test}

PASAT is a test in which the subject listens to two single-digit numbers from an audio device, adds them, and answers the sum verbally. Two sets of sixty numbers are given to the subject. The first set is presented with two-second intervals between each number, and the second set is presented with one-second intervals. These two sets are designated as 2-sec Test and 1-sec Test, respectively. 1-sec Test is more difficult than 2 -sec Test because the interval between the numbers is shorter. In order to evaluate the subjects' answers, the operator records their answers on the evaluation form (Fig. 5).

\subsection{Glucose Sensor}

The glucose sensor we used is the NIPRO Care Fast C (Fig. 6). This device was chosen because it can measure blood sugar easily, safely, and quickly so that subjects could take their own blood samples. The device uses the enzyme electrode method to measure glucose based on amperometric method. Its error range is less than $\pm 20[\%]$ for BSLs higher than the known concentration of 75 [mg/dL]. Though its error range is not small, the tendency of blood sugar change can be evaluated by the sensor.

\section{Modelling Technique}

Since there are a comparatively large number of components in hemoglobin measurements by NIRS,

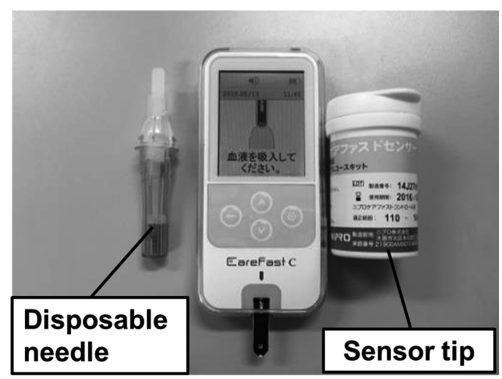

Fig. 6 Glucose sensor [NIPRO Care Fast C] by NIPRO

a data reduction step is required for this method. This study used the hierarchical decomposition analysis, HDA [8], which consists of the principal components analysis, PCA, and multivariable autoregressive (MAR) modelling technique. HDA reconstructs the estimated parameters into a hierarchical structure or an upper triangular form. The HDA is explained in section 3.1.

\subsection{Principal Components}

Data vector $X$ is constructed using all the observed measurements, noted by $\left\{x_{i}(n): i=1, \cdots, M, n=\right.$ $1, \cdots, N\}$ as in equation (1) in this paper, and it is decomposed by PCA as in equation (2) to reduce the number of components.

$$
\begin{aligned}
& X=\left[\begin{array}{cccc}
x_{1}(1) & x_{1}(2) & \ldots & x_{1}(N) \\
x_{2}(1) & x_{2}(2) & & \vdots \\
\vdots & & \ddots & \vdots \\
x_{M}(1) & \ldots & \ldots & x_{M}(N)
\end{array}\right] \\
& X=C^{T} W T
\end{aligned}
$$

In equation (2), $C$ is a spatially weighted matrix, $W$ is a diagonal matrix of eigen values, and the resul$\operatorname{tant} T$ is a matrix of principal components. This step changes the data matrix $X(M \times N)$ to smaller sized matrix $T(P \times N)$, where $N$ is the number of measurements (data length), $M$ is the number of original channels, $P$ is the number of channels after they have been reduced by a certain level of contribution rate, denoted in this paper by $M^{-1}$.

\subsection{Multivariable Autoregressive Model}

As stated in 3.1, each row in $T$ describes the timeseries data of the principal components. In the second step, the rows of $T$ are modelled using an autoregressive technique. Hierarchical decomposition is one of the MAR modelling techniques that turns its parameters into a hierarchical structure. The first step is PCA, as stated in section 3.1. Then, the MAR model (3) of the temporal components $T_{p, n}$ is determined by minimizing the sum of the squared residual values (4). 


$$
\begin{aligned}
& T_{p, n}=R_{p, n}+\mu_{p}+\sum_{q=1}^{P} \sum_{l=1}^{L} A_{q, p, l} T_{q, n-l} \\
& R_{M L A R}=\sum_{p=1}^{P} \sum_{n=1}^{N} R_{p, n}^{2}
\end{aligned}
$$

In $(3), \mu_{p}$ denotes a mean value, $R_{p, n}$ denotes a residual value and $A$ denotes a three-dimensional matrix with estimated model parameters given by the Yule-Walker equation. $L$ is the model order estimated by AIC.

The above steps give us an MAR model, but the model is not unique, which is resolved by the following procedure.

The first step is to seek a transformation that is able to orthonormalize the innovations $R_{p, n}$. If we denote the transformation matrix as $K$, new temporal components and estimates are given by the following equations:

$$
\begin{aligned}
& T^{\prime}=K T \\
& A_{l}^{\prime T}=K A_{l}^{T} K^{-1}
\end{aligned}
$$

The above transformation does not completely resolve the non-uniqueness problem. To proceed further, a rotation must be found whose transformed temporal components are consistent with the hierarchical structure. If $Q$ is denoted as transformation matrix, $Q$ is obtained iteratively via a procedure similar to the Jacobi matrix diagonalisation.

Finally, HD components are chosen by using the transformation $Q$ as shown in equations (7) and (8).

$$
\begin{aligned}
& T_{H D}=Q T^{\prime} \\
& A_{H D}^{T}=Q A_{l}^{\prime T} Q^{-1}
\end{aligned}
$$

When the HD components are estimated, the upper triangular elements of the estimated parameters are weights for feed-forward interactions and the lower triangular elements characterize feedback interactions. The diagonals are the self-driving weights describing the contribution to itself.

If $T_{H D}$ represents the HD components determined from the data vector $X$, equation (9) is derived from equations (2), (5), and (7).

$$
X=C^{T} W K^{-1} Q^{-1} T_{H D}
$$

$X$ is an observable signal and $T_{H D}$ denotes unobservable basic generators. If we apply $X$ as cerebral hemodynamic signal, then $C^{T} W K^{-1} Q^{-1}=G$ can be considered as representing a transfer function from basic generators to the cerebral oxygenation signal, in a sense, a brain mapping function for the task.

\section{Experimental Results}

\subsection{Blood Sugar Levels}

The averages of BSL are shown in Fig. 7. BSL increases gradually after the intake of glucose (red line). However, it shows a tendency to decrease after the test. It can be assumed that the brain uses glucose during the test.

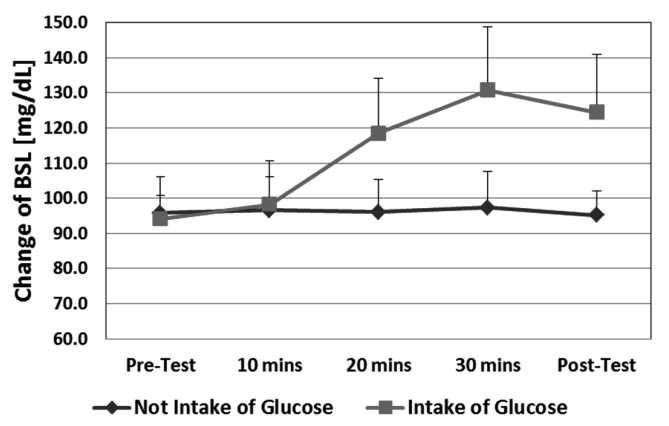

Fig. 7 The averages of BSL in all subjects

\subsection{Paced Auditory Serial Addition Test}

The results indicating correct subject answer rates in the PASAT are shown in Fig. 8. Although there were 14 subjects, there are 21 in total sets of data because some subjects participated in both experiments, WOT-100 and ETG-7000 (Table 1). The different difficulty levels of both tests are described in the frequency distribution of the correct answer rate histogram. The average score of the 2 -sec Test was $84.2 \pm 2.9 \%$ (Not intake of Glucose: NG) and 90.3 $\pm 2.2 \%$ (Intake of Glucose: IG). The average score of the 1 -sec Test was $59.7 \pm 4.1 \% \quad(\mathrm{NG})$ and 70.5 $\pm 3.8 \%$ (IG) . Thus, the average 2 -sec Test score was higher than the score of the 1 -sec Test and a statistically significant difference $(\mathrm{p}<0.05, \mathrm{~T}$-test $)$ was confirmed between them. Furthermore, there was a statistically significant difference between NG and IG test scores, as well.

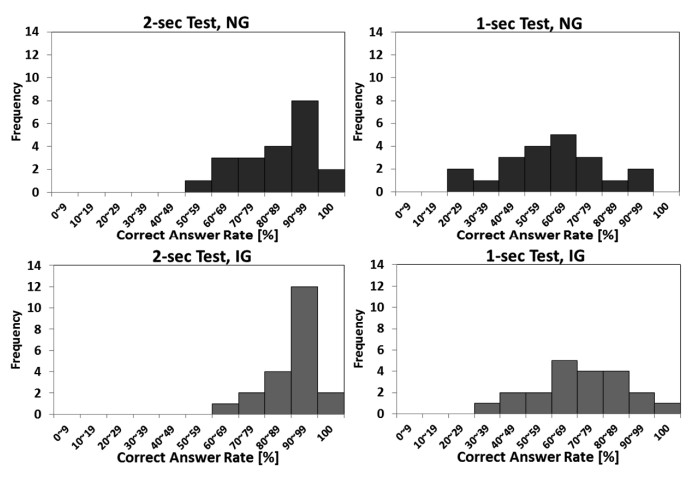

Fig. 8 Correct answer rates of PASAT

Table 1 The experiments which subjects completed \begin{tabular}{|c|c|c|c|c|c|c|c|c|c|c|c|c|c|c|c|}
\hline Subject & A & B & C & D & E & F & G & H & I & J & K & L & M & N & O \\
\hline \hline
\end{tabular}

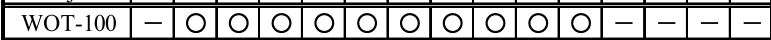
\begin{tabular}{|l|l|l|l|l|lllllllllll}
\hline ETG-7000 & - & 0 & - & 0 & 0 & 0 & 0 & - & 0 & 0 & - & 0 & 0 & 0 & 0 \\
\hline
\end{tabular} 

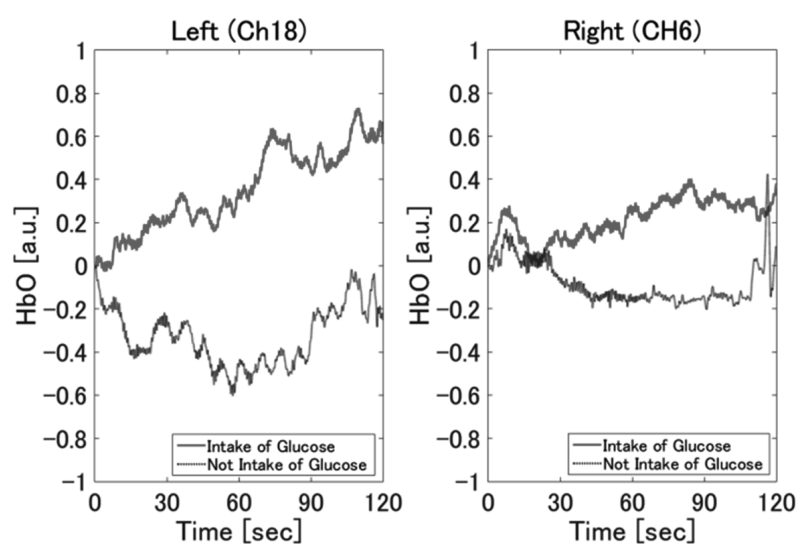

Fig. 9 The change of HbO during 1-sec Test by WOT100

\subsection{Change of $\mathrm{HbO}$}

The averaged $\mathrm{HbO}$ changes are shown in Fig. 9 and Fig. 10, calculated by taking the collective mean of the data. In $\mathrm{CH} 8,17$, and 18 of WOT-100, HbO averages increased significantly after taking glucose. In $\mathrm{CH} 16$ of probe 1 (the right) and $\mathrm{CH} 2,9$ and 15 of probe 2 (the left) of ETG-7000, HbO averages showed a significant increase after taking glucose as well.

CH17, 18 (WOT-100), and CH9 (ETG-7000) are on the left side of the brain; they seem to be located in the area called the dorsolateral prefrontal cortex (DLPFC). DLPFC takes charge of difficult cognitive tasks involving arithmetic processing, verbal working memory, and attention. The authors consider that DLPFC was activated to use more energy (i.e., glucose) in response to the large load of the 1-sec Test.

Based on these results, the authors selected the channel relating to the DLPFC area. The HbO average of the DLPFC area shown by right channels $(\mathrm{CH}$ 5,7 , and 8) and left channels (CH14, 16, and 17) using the WOT-100 is shown in Fig. 11. Furthermore, the $\mathrm{HbO}$ average in the DLPFC area shown by right channels ( $\mathrm{CH} 5$ and 14) and left channels (CH9 and 18) using the ETG-7000 is shown in Fig. 12. The HbO average at channel 9 during the 1-sec Test changed significantly when compared to the 2 -sec Test $(\mathrm{p}<$ 0.05). The authors assumed that the difficult task (1sec Test) related to left prefrontal cortex near the left DLPFC.

\subsection{Analysis by HDA}

The HbO data were treated by the other data. The method used in this paper is the hierarchical decomposition analysis method, HDA, which is explained in section $\mathbf{3}$.

Fig. 13 shows results of the first step of HDA. If the threshold of $1 / 14$ is applied, there are two principal components. By summarizing the results of all test subjects, selected components can be decreased to a range from 1 to 6 for ETG-7000 (44 channels) and 1 to 3 for WOT-100 (14 channels). The averages are shown in Fig. 14 and Fig. 15.
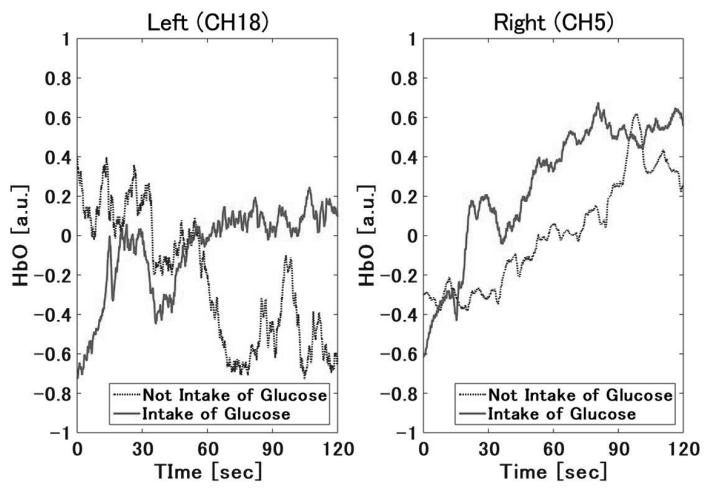

Fig. 10 The change of HbO during 1-sec Test by ETG7000

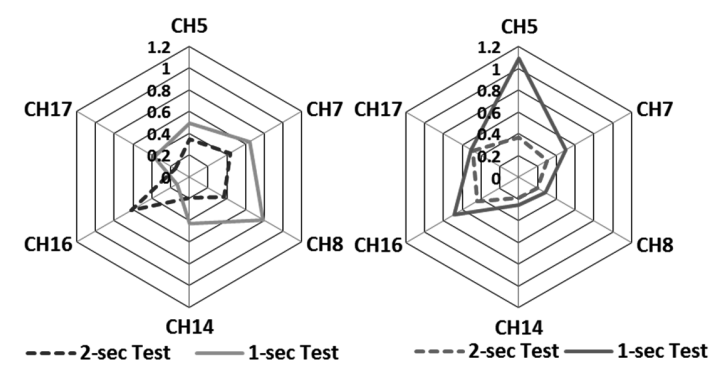

Fig. 11 The HbO average during PASAT as measured by WOT-100

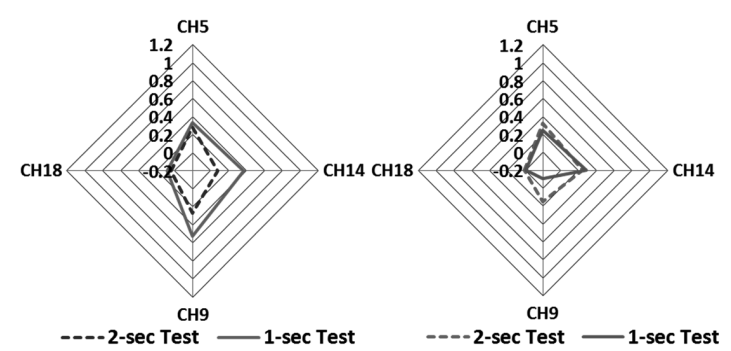

Fig. 12 The $\mathrm{HbO}$ average during PASAT as measured by ETG-7000

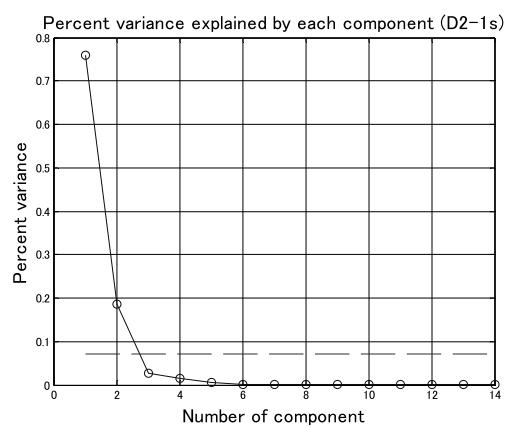

Fig. 13 A first step of HDA, percent variance

The number of principal components after tests with glucose intake tends to be smaller than that of tests without glucose intake. Particularly for data obtained using the WOT-100, a significant difference exists for the 1sec-Test. These results suggest that the 
The Average of Principal Components, ETG

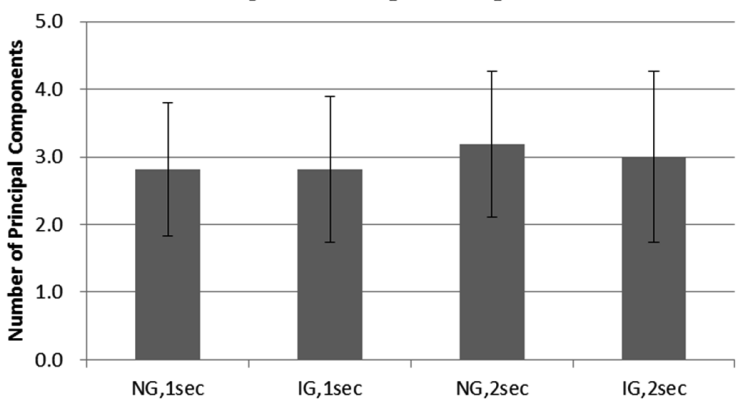

Fig. 14 The average of principal components (ETG)

The Average of Principal Components, WOT

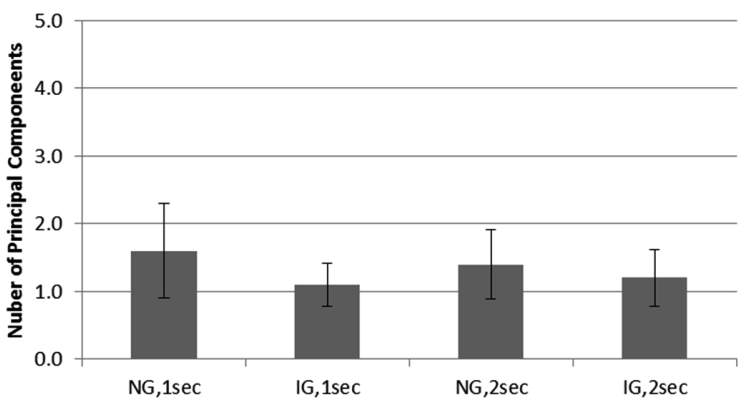

Fig. 15 The average of principal components (WOT)

active area, typically in the frontal lobe, is reduced by glucose ingestion.

HDA were applied for estimating MAR form models. The variance resulting from errors between the original signal and reconstructed one is shown in Fig. 16, which indicates some channels that are important to the task. Channels on the left side (WOT-100) and the front side (ETG-7000) tend to have small errors.
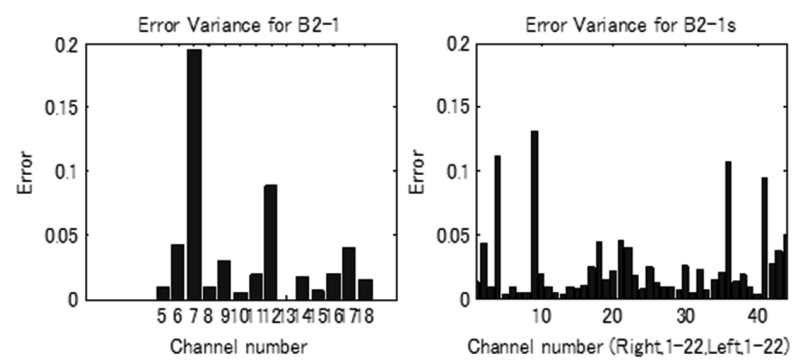

Fig. 16 Error variance by PCA and HDA

As for the order of the estimated models, the order average is summarised in Fig. 17 and Fig. 18. ETG-7000 produces a model order of approximately 2 , whereas WOT-100 produces an order of 2 to 3 . If the sampling rates are taken into account, blood flow of the frontal lobe has more influence than that of the temporal lobe. Moreover, order, as measured by the WOT-100 becomes smaller after taking glucose, which shows the effects of glucose on this kind of attentional task.

A final result of the HDA analysis is presented in
The Average of Estimated Model Order, ETG

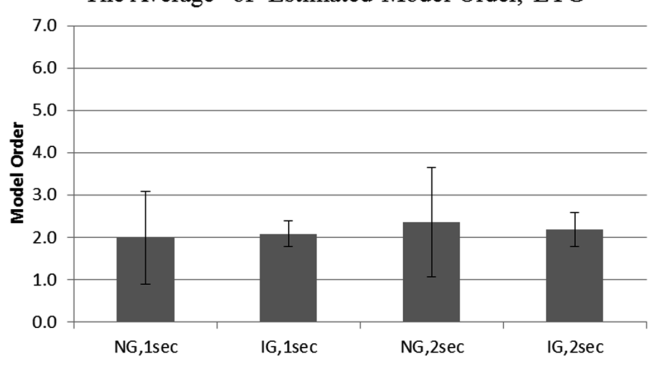

Fig. 17 The average of order (ETG)

The Average of Estimated Model Order, WOT

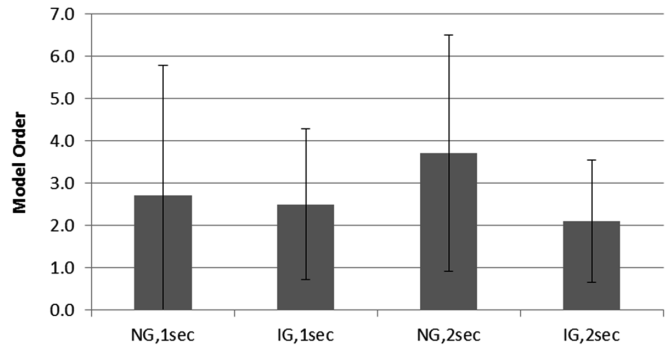

Fig. 18 The average of order (WOT)
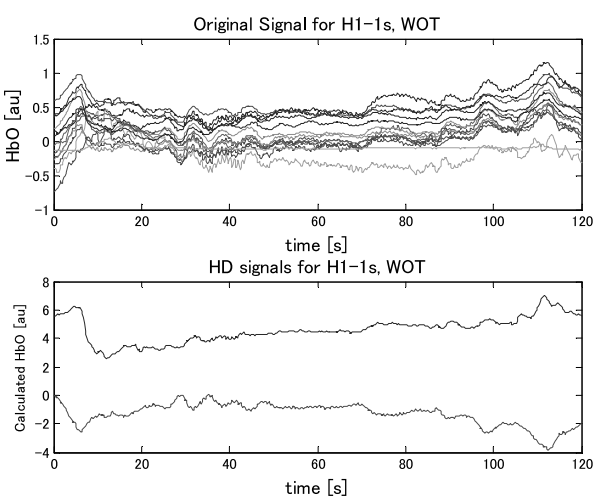

Fig. 19 Original and HD signals

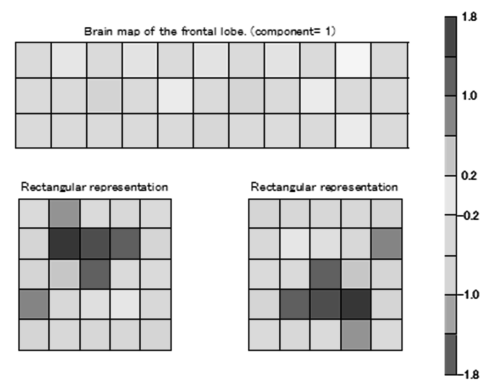

Fig. 20 Estimated brain map for subject E, 2-sec

Fig. 19, showing the original $\mathrm{HbO}$ signals (upper) and the hierarchically decomposed ones (lower). A brain map can be created [9] by the estimated model for each subject from HDA which denotes the weight from the inner signal to the measured $\mathrm{HbO}$ in the cerebral cortex (Fig. 20). 


\section{Conclusions}

This paper discussed hemodynamics in both the prefrontal and temporal cortex during attentional control task. Correct answer rates increased significantly after glucose intake compared to the rates before glucose intake. Blood flow measurements changed according to the test difficulty at the left DLPFC as measured by both WOT-100 and ETG-7000, indicating that the task activated the attention function. Because BSLs decreased after the PASAT was performed, it is thought activation of the working memory was enhanced by glucose intake, and there was concentration of the blood $(\mathrm{HbO})$ in the left DLPFC. Analysis by HDA and the derived brain map suggested that the same areas were dominant for the kind of attentional tasks addressed in this paper.

\section{References}

[1] R. Takizawa and M. Fukuda: Functional neuroimaging as a laboratory test and the first Advanced Medical Technology in Psychiatry; MEDIX, Vol. 53, pp. 30-35 (2010)

[2] J. Ponsford and G. Kinsella: Attentional deficits following closed-head injury; J Clin Exp Neuropsychol, Vol. 14, No. 5, pp. 822-838 (1992)

[3] H. Sakai and T. Kouchiyama: Difficulty levels in a PASAT and the detection of activated regions in the brain; The Japanese Journal of Occupational Therapy, Vol. 48, No. 12, pp. 1255-1262 (2014)

[4] N. Aizawa, C. Uchiyama, Y. Nakamura, K. Makita, M. Ishibashi and M. Iwakiri: Measuring prefrontal hemodynamic changes during cognitive tasks: A review on NIRS studies; School Crisis and Mental Care, Vol. 2, pp. 59-72 (2011)

[5] W. Kuschinsky, S. Suda and L. Sokoloff: Am. J. Phys-iol., 241, pp. H772-H777 (1981)

[6] P. T. Fox, M. E. Raichle and M. A. Mintun: Science, 241, pp. 462-464 (1988)

[7] M. Haida: Implication of a signal from brain optical topography; MEDIX, Vol. 36, pp. 17-21 (2002)

[8] M. A. Repucci, et al.: General strategy for hierarchical decomposition of multivariate time series: Implications for temporal lobe seizures; Annals of Biomedical Engineering, Vol. 29, pp. 1135-1149 (2001)

[9] K. Oura: A study on brain mapping technique based on hierarchical decomposition analysis; IEEJ Trans.EIS, Vol. 129, No. 9, pp. 1675-1681 (2009)

\section{Authors}

Kanako Mikami

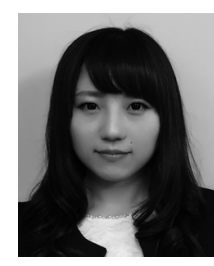

Kanako MIKAMI was born on 15 th Oct. 1990. She received B.E. and M.E degree from Kokushikan University in 2013 and 2016. Presently, she is a graduate student of Doctoral course majoring in the Applied Systems Engineering in Kokushikan University. Her research fields include the higher-order brain function in the human, using NIRS, MRI, EEG, and so on. She has the license of Health Information Manager (HIM) from Council of Four Hospital Organizations, Japan.

\section{Kunihiko Oura (Member)}

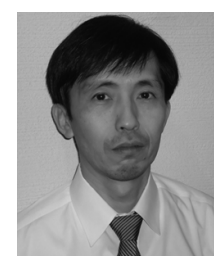

Kunihiko OURA was born on 22nd February, 1969. He received his B.E.,M.E. and D.E. degrees from Waseda University, Japan, in 1991,1993, and 1997. He is currently a professor in the Department of Health and Medical Engineering, School of Science and Engineering, in Kokushikan University, Japan. His research interests include bio-signal modelling, brain functional imaging and stochastic system analysis. 